\title{
Julia and Hans Weertman: A legacy of scholarship, mentorship, and lives well-lived
}

\author{
By Sonal Rangnekar and Riley Hanus
}

Conducting careful experiments, creating a "builder culture," and making good, well-characterized samples that were shared with collaborators around the globe. These were part of Julia Weertman's MO, as described by former graduate student Paul Sanders (now the Patrick Horvath Endowed Associate Professor of Materials Science and Engineering at Michigan Technological University) at the Symposium in Honor and Remembrance of Johannes (Hans) and Julia Weertman, held November 16 in Evanston, Ill. The
Weertmans' eagerness to publish and their perception of publishing as a dialogue, their stature as role models, and their mentorship of others were common themes among the many speakers honoring them at the event.

Friends, family, former students, and colleagues traveled from as far away as Japan and Switzerland to share their fond remembrances of Julia and Hans. Technical talks highlighted their contributions to materials science, particularly dislocation theory and the mechanical

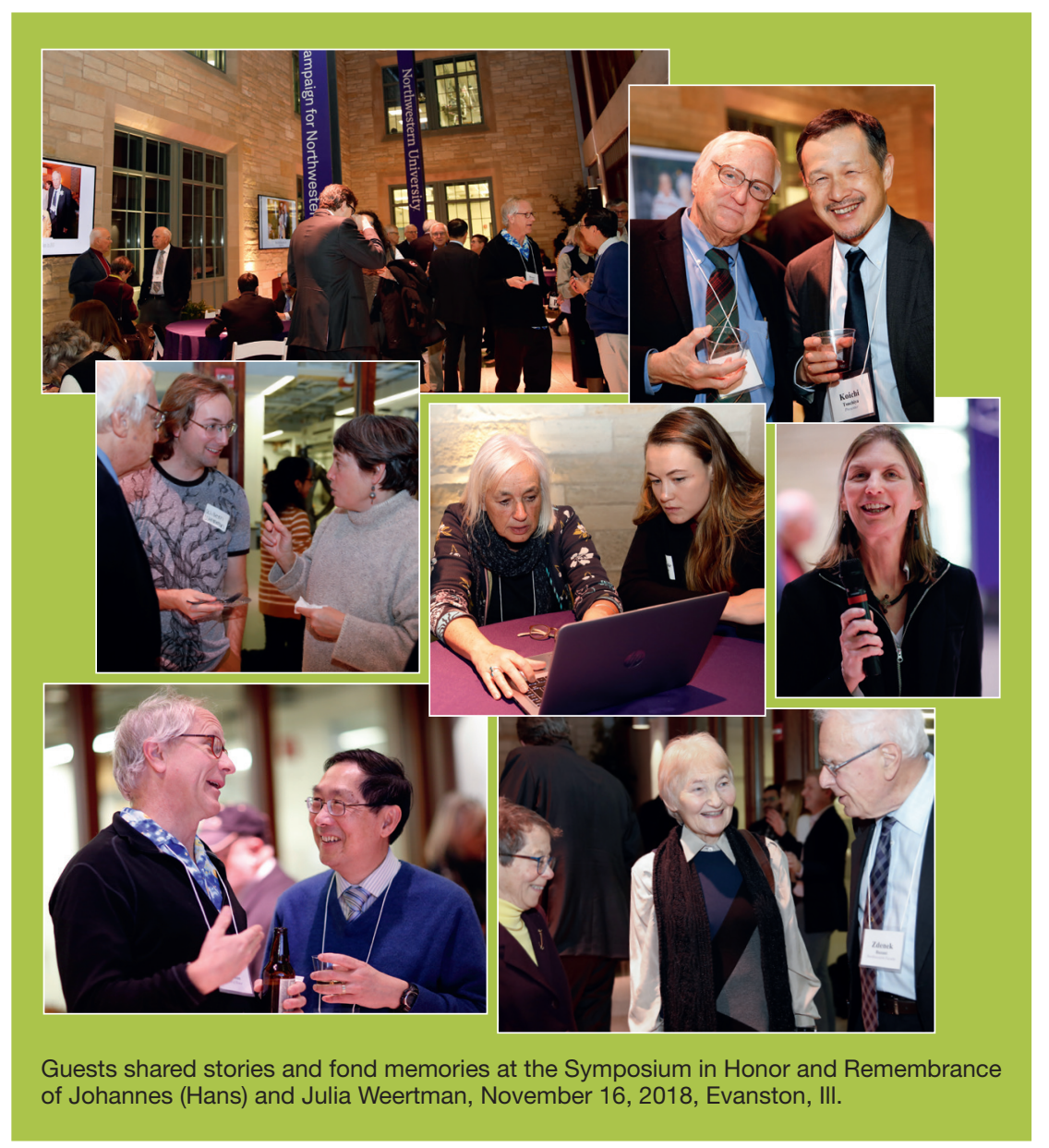

properties of metals, but also geophysics and glaciology. Many speakers described themselves as part of the Weertmans' extended family, connected by experimental results and holiday cards depicting Santa Fe. In addition to discussions about science, students and colleagues remembered group dinners at the Weertmans' residence, trips to Doc Watson concerts and Chicago jazz bars, and social visits that continued long after their theses had been signed.

From these anecdotes emerged a picture of MRS Fellow and Von Hippel awardee Julia Weertman that was vivid enough for graduate students who had never met her to be in awe of her personal fortitude and professional accomplishments. Julia modestly described the 1960s as a period when she focused on "raising our two children," though she co-authored several seminal papers with Hans and published their popular textbook, Elementary Dislocation Theory, which has become an indispensable text in the field of materials microstructure.

Taking it upon herself to be a guiding light for younger women in science, Julia recruited and trained a large number of female students. She adeptly navigated the male-dominated scientific community, learning to reject secretarial positions on scientific committees and instructing her female protégés to avoid leadership that would only lead to "Service Awards."

Carolyn Aita, distinguished professor emeritus, University of WisconsinMilwaukee, remembered Julia's “inescapable" mentorship. She was the go-to person for professional decisions, and her story empowered her students to balance family and career. According to Bill Nix, Stanford University, she became a mentor for his female students. Julia commonly 
sent handwritten notes to prospective students to encourage them to apply to the graduate program. Her enthusiastic mentorship persisted late into her life. If she asked you to deliver a last-minute keynote pres-entation about an unfamiliar topic on her behalf, you could expect Julia to send you revised presentation slides from her hospital bed until you felt inspired to present. As Andrea Hodge, University of Southern California, observed, Julia treated her students as equals, but required them to work hard to prove themselves.

The hard work that Julia and her students put into novel and high-quality research has resulted in countless scientific papers and patents, dating back to the 1950 s, which remain highly cited and relevant to this day. Her pioneering work spans fields from nanocrystalline materials synthesis and characterization, to the study of materials microstructure and defects using neutron and synchrotron x-ray scattering, to magnetic properties. Julia's collaborators, such as Jeffery Eastman, Peter Jemian, and Gabrielle Long, from Argonne National Laboratory, recalled how experiments performed in their homebuilt synthesis chambers in the 1990s continue to influence state-ofthe-art science. Her research has laid the foundation for understanding nanoscale energy materials, engineering ceramics, catalysts, and more. Despite experiencing great success with fundamental science, Julia remained ever-pragmatic and aimed to find technological applications of nanocrystalline materials. Her collaborator, Helena Van Swygenhoven-Moens, professor at the Paul Scherrer Institute and École Polytechnique Fédérale de Lausanne in Switzerland, described how pleased Julia would be to know of recent applications of nanocrystalline alloys in mechanical components of consumer products and stents for cardiovascular surgeries.

While Julia enjoyed hands-on experimental research, Hans Weertman saw the world through mathematics. Encouraging his students, such as David Cole, Dartmouth College, to "worry about mechanisms, not scale," Hans's work enabled fundamental understanding of creep in crystalline materials, from metallic alloys to the ice of glaciers, and slip of earthquake faults. Hans's contributions in this area were honored with the naming of Weertman Island in Antarctica. Hans spent some of his early career elucidating dislocation mechanics in icea zeitgeist of a topic that, according to Douglas MacAyeal, The University of Chicago, captivated some of the best scientific minds post-World War II. Hans shared Julia's commitment to improving diversity in science and to giving credit where due. He encouraged students in his advanced dislocations class to publish their class projects and acknowledged their help proofreading his "DislocationBased Fracture Mechanics" text.

After listening to the stories about Julia and Hans Weertman, it is clear that they were a rare couple: phenomenal scientists who set high standards for their students in a warm supportive environment. Their legacy, like their science, will stand the test of time. It lives on in their children, Bruce and Julia, their students and colleagues, and in the current and future students of materials science and engineering, who can be inspired by their example.

\section{3th New Diamond and Nano Carbons Conference to be held in Taiwan} ndnc2019.org

$\mathrm{T}_{\mathrm{c}}^{\mathrm{h}}$ 13th New Diamond and Nano Carbon Conference (NDNC 2019) will be held in Taiwan, May 12-17. The conference will present recent breakthroughs in the synthesis, physics, and applications of diamonds and other carbon nanostructures, such as graphene and nanotubes.
The program features four plenary talks (Huan-Cheng Chang, Academia Sinica, Taiwan; Toshiaki Enoki, Tokyo Institute of Technology, Japan; Ho-Kwang Mao, HPTSTAR, China, and Carnegie Institution, USA; and Boris Yakobson, Rice University, USA), 25 invited talks, oral contributions, and poster sessions.
NDNC is a merger of the former International Conference on New Diamond Science and Technology (ICNDST), initiated in 1988 in Japan, and the International Conference on Applications of Diamonds (Applied Diamond Conference), initiated in 1991 in the United States. For more information, visit ndnc2019.org.

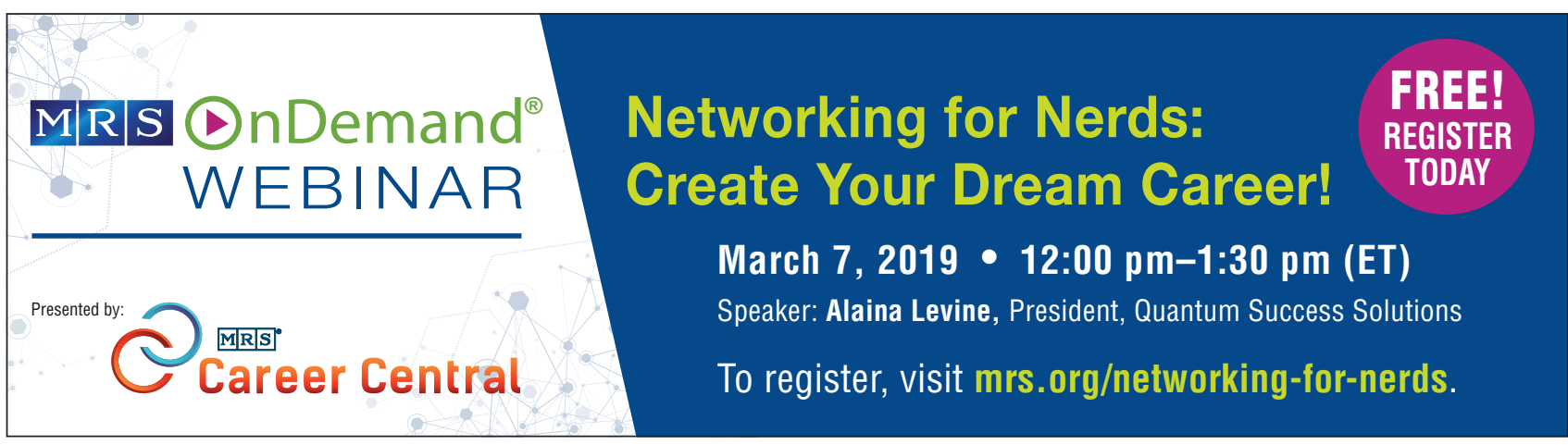

